\title{
Bubbling and Frothing Liquids
}

\author{
Paul W. Cleary \\ CSIRO, Australia \\ paul.cleary@csiro.au
}

\author{
Soon Hyoung Pyo \\ ETRI, South Korea \\ shpyo@etri.re.kr
}

\author{
Mahesh Prakash \\ CSIRO, Australia \\ mahesh.prakash@csiro.au
}

\author{
Bon Ki Koo \\ ETRI, South Korea \\ bkkoo@etri.re.kr
}

\begin{abstract}
We present a discrete particle based method capable of creating very realistic animations of bubbles in fluids. It allows for the generation (nucleation) of bubbles from gas dissolved in the fluid, the motion of the discrete bubbles including bubble collisions and drag interactions with the liquid which could be undergoing complex free surface motion, the formation and motion of coupled foams and the final dissipation of bubbles. This allows comprehensive simulations of dynamic bubble behavior. The underlying fluid simulation is based on the mesh-free Smoothed Particle Hydrodynamics method. Each particle representing the liquid contains an amount of dissolved gas. Gas is transferred from the continuum fluid model to the discrete bubble model at nucleation sites on the surface of solid bodies. The rate of gas transport to the nucleation sites controls the rate of bubble generation, producing very natural time variations in bubble numbers. Rising bubbles also grow by gathering more gas from the surrounding liquid as they move. This model contains significant bubble scale physics and allows, in principle, the capturing of many important processes that cannot be directly modeled by traditional methods. The method is used here to realistically animate the pouring of a glass of beer, starting with a stream of fresh beer entering the glass, the formation of a dense cloud of bubbles, which rise to create a good head as the beer reaches the top of the glass.
\end{abstract}

CR Categories: I.3.5 [Computer Graphics]: Computational Geometry and Object Modeling-Physically based modeling; I.3.7 [Computer Graphics]: Three-Dimensional Graphics and RealismAnimation

Keywords: Natural phenomena, fluid dynamics, smoothed particles hydrodynamics, discrete element method, bubbles

\section{Introduction}

Liquids are an essential substance in our daily life and have attracted the attention of many researchers. The behavior of different liquids in their various states has been the subject of numerical simulation for around three decades now. Recent developments in computer graphics has enabled this understanding to be applied to the production of films, animations and commercials.

Many of these research results have successfully improved the quality of liquids presented in various applications. However, there are still many areas where the lack of realism leads to a need for artistic and therefore labor intensive methods to make the liquids appear realistic. For example, a real liquid has many features such as foam, spray and bubbles associated with it.

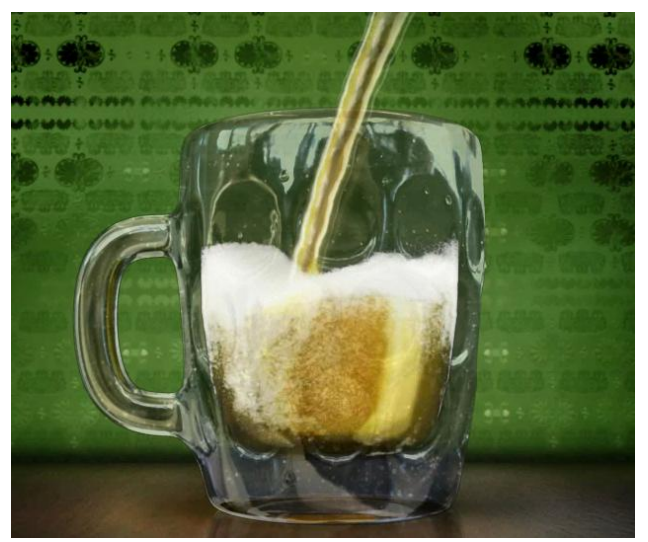

Figure 1: Bubbles in beer

These features are still not automatically created by fluid simulators. In this paper, we focus on the automatic generation of bubbles based on the physics of dissolved gas diffusion and nucleation and their interaction with liquids for application in carbonated drinks, such as beer, ale, soft drinks and champagne. An example of the foaming of beer as it is poured is shown Figure 1. This is one of the popular targets for making commercials.

In the proposed method each bubble is described as a discrete entity that interacts with the surrounding liquid and moves based on buoyancy, cohesion and drag forces imposed by the liquid and surrounding bubbles. This in turn gives the simulated results highly realistic behavior.

\section{Related Work}

\subsection{Particle based Fluid Simulation}

Significant progress has been made since the introduction of the full Navier-Stokes equations in computer graphics for realistic animation of fluids [Kajiya and Herzen 1984]. Stam [1999] presented a semi-Lagrangian approach to improve the performance of fluid animations by using large time steps for the simulation. Foster and co-workers introduced and improved the level-set method to track the surface of water for better appearance [Foster and Fedkiw 2001; Enright et al. 2002]. These works became the state-of-the-art of fluid simulation in computer graphics.

As an alternative to Eulerian fluid methods, particle based fluid simulation techniques have long been used in simulation of complex free surface flows for industrial and geophysical flows. A powerful Lagrangian method is Smoothed Particle Hydrodynamics (SPH). It was originally developed by Gingold and Monaghan [1977] for astrophysical applications and was extended to the simulation of incompressible fluid flows interacting with boundaries by Monaghan [1992; 1994]. SPH is a particle based method well adapted for modeling coupled fluid flows, solid structure deformation and heat transfer. The particles represent volumes of discretized fluid or solids that move around in response to the fluid or solid stresses produced by interaction with other particles. Due to its Lagrangian nature SPH does not use grids or meshes to track the fluid and calculate the fluid velocities. The SPH particles carry all the computational information and they are free to move. The Lagrangian nature 
of SPH means that the particles will automatically follow complex flows. SPH was first used in industrial flow modeling applications such as casting by Cleary [1998] and for large scale geophysical applications [Cleary and Prakash 2004]. Since its introduction SPH has been used for a broad range of complex multi-physics flow simulations, see reviews by Monaghan [2005] and Cleary [2007].

In computer animation the SPH technique was first introduced by [Desbrun and Gascuel 1996] for simulating highly deformable bodies. More recently SPH has been used in computer graphics by Muller et al. as a solution for the animation of multiple fluids such as air and water [Muller et al. 2003; Muller et al. 2005]. Their original intention for adopting SPH to computer graphics was for interactive applications. Premoze et al. [2003] also introduced the SPH method around the same time as Muller's group for simulating fluid flow in computer graphics involving free surface behavior, fragmentation and break-up . Thürey et al. [2006] presented a hybrid shallow water and particle based Lattice Boltzmann method for simulating open water phenomena. The present paper is also based on SPH for fluid simulation.

More recent research has focused on increasing the realism of simulated fluids by adding details such as foam, bubbles, and spray. Takahashi et al. [2003] integrated particle systems into Cubic Interpolated Propagation based fluid solver to create foams and splashes on the simulated fluids. They implemented a rule based system for the generation, disappearance and transition of splashes and foam. Kim et al. [2006] presented splashing water animations using a particle level set technique by representing massless marker particles escaping from water as splashes. A method to simulate a variety of small scale fluid phenomena such as water drops on surfaces by enforcing contact angles at the intersection of fluid free surface and solid objects was presented by Wang et al. [2005]. Geiger et al. [2006] used a particle level set method and secondary particles to simulate realistic fluids with foam and mist for "Poseiden". Simulation of bubbles is also a step in the direction of creating more realistic fluid animation.

\subsection{Bubbles}

The main sources of bubbles in liquids are:

- trapped air pockets created during complex liquid motion,

- gas dissolved in the liquid,

- bubbles generated during phase change (e.g., boiling).

There has been some research towards modeling bubbles from trapped air pockets and boiling, but little work has been done in terms of simulating bubbles from gas contained in liquids in Computer Graphics. Hong and Kim [2003] combined the Volume-ofFluid (VOF) and front-tracking methods to create bubbles emanating from entrapped air pockets and handled the topological changes of bubble shapes using surface tension. Greenwood and House [2004] created bubbles using level-set marker particles and trapped air pockets, but no consideration was given to change of bubble shape. Song et al. [2005] created bubbles by modeling multiphase fluids and converting potentially dissipative air cells into bubbles. Muller et al. [2005] used a particle based approach to simulate multiple fluids and dynamically generated air particles to represent bubbles emanating from the entrapped air pocket. They also presented an example of creating bubbles from the change of temperature by boiling water. Mihalef et al. [2006] proposed a method to couple gas and liquid with variable temperature and mass transfer for physics-based boiling simulations.

One of the main issues for bubbles is how to construct the internal structure formed at the interface between multiple bubbles. Kuck et al. [2002] presented a method to simulate bubbles using spring forces and constructed thin interfaces among bubbles for rendering them more effectively. Later, Greenwood and House [2004] adopted their method into the simulation of fluids to model bubbles emanating from trapped air pockets. A regional level set method to track multi-manifold surfaces for thin bubble films was used by Zheng et al. [2006] with a semi-implicit surface tension model to compute the shape of bubbles efficiently. This issue is an important one to deal with for the exact modeling of bubble interaction.

Most research in the area of bubble formation has dealt with a small number of discrete bubbles large in size (volume) in comparison with the liquid volume. In this paper we address cases with large numbers of discrete bubbles which are small to medium in size in comparison with the liquid volume. The method is able to handle all aspects of bubble generation, transport (coupled to the fluid phase), foam formation and flow and bubble dissipation. Applications include representation of bubbles and foam mainly in carbonated liquids such as beer, carbonated drinks and champagne.

\section{Coupled Discrete Bubble - Fluid Dynamics}

The physics of bubble creation in carbonated liquids is relatively well understood. Liquids such as beer and champagne contain dissolved gas, typically carbon dioxide $\left(\mathrm{CO}_{2}\right)$. In the case of beer in a capped bottle, $\mathrm{CO}_{2}$ dissolved in the beer is in equilibrium with the $\mathrm{CO}_{2}$ gas in the free neck of the bottle. When the bottle is uncapped, the gas in the neck escapes and the equilibrium is broken. As the beer is poured into a glass, the super-saturated solution contacts nucleation defects in the glass surface which creates tiny bubbles in the beer. These detach from the nucleation sites and rise buoyantly within the liquid. Further bubbles are created in streams from the glass defects at rates controlled by the rate of diffusive and convective gas supply to this location. This depends on the amount of gas dissolved in the liquid and the motion of the liquid near the glass surface. Realistic gas generation is most easily accomplished by including all the physics of liquid phase gas transport and nucleation.

\subsection{Bubble collisions}

Small and medium sized bubbles are relatively well approximated as discrete entities with fixed shapes (most easily spherical). We use a soft particle discrete element style method here [Cleary 2004] to model the collisional interaction of the discrete bubbles with each other and with other solids and boundaries. We model the normal component of the force using a linear spring and dash pot or damping model in Figure 2.

$$
F_{n}=-k \Delta x+C v_{n}
$$

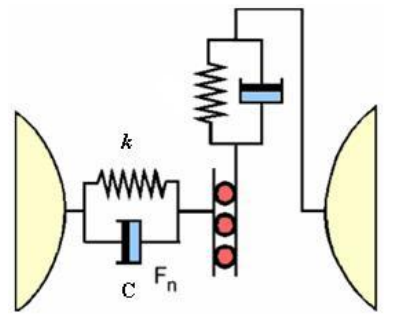

Figure 2: Schematic of spring dashpot model

where $\Delta x$ is the overlap between the bubbles or the bubble and a solid surface, $v_{n}$ is the normal component of the relative velocity, $k$ is the spring constant and $C$ is a suitable damping constant. There is little shear interaction between bubbles because of the fluid layer in between, so no shear component of the force is used for wet foam. The normal force is directed along the line between the centers of the bubbles. In the above equation if $\Delta x>0$ then we have collisional forces: 


$$
\mathbf{F}_{\text {coll }}=\mathbf{R F}_{\mathbf{n t}}
$$

where $\mathbf{F}_{\mathbf{n t}}=\left(\begin{array}{c}F_{n} \\ 0 \\ 0\end{array}\right)$ and $\mathbf{R}$ is any 3D rotational matrix from the world coordinate system to the collision frame defined with the bubble contact normal being in the $x$-direction in the collisional frame.

\subsection{Bubble fluid interaction}

Bubbles are positively buoyant because of the large density difference between the bubble and the water that it displaces. The buoyancy $\mathbf{F}_{\mathbf{b}}$ is just given by the weight difference and depends on the diameter of the bubbles as presented below:

$$
\mathbf{F}_{\mathbf{b}}=\mathbf{g} V_{b u b}\left(\rho_{b u b}-\rho_{l i q}\right)
$$

where $\mathbf{g}$ is gravity, $V_{b u b}$ is the bubble volume, $\rho_{b u b}$ is the bubble density and $\rho_{l i q}$ is the density of the background liquid.

The bubbles are coupled to the liquid phase via a suitable empirical drag law. For the present examples we use a simple drag force $\mathbf{F}_{\mathbf{d}}$ for isolated bubbles in a large volume of fluid. The drag force has two forms dependent on the bubble Reynolds number:

$$
\operatorname{Re}_{b u b}=\frac{2 \rho_{\text {liq }}|| \mathbf{v}_{\mathbf{b u b}}-\mathbf{v}_{\mathbf{l i q}} \| r_{b u b}}{\mu_{l i q}}
$$

where $r_{b u b}$ is the bubble radius and $\mu_{l i q}$ is the liquid viscosity.

- For $R e_{b u b}<1.0$ or high viscosity regime (Stokes law)

$$
\mathbf{F}_{\mathbf{d}}=-6 \pi r_{b u b} \mu_{l i q}\left(\mathbf{v}_{\mathbf{b u b}}-\mathbf{v}_{\mathbf{l i q}}\right)
$$

- For $R e_{b u b}>1.0$

$$
\mathbf{F}_{\mathbf{d}}=-0.5 C_{d} \rho_{b u b} A_{b u b}\left(\mathbf{v}_{\mathbf{b u b}}-\mathbf{v}_{\mathbf{l i q}}\right)\left\|\mathbf{v}_{\mathbf{b u b}}-\mathbf{v}_{\mathbf{l i q}}\right\|
$$

where $C_{d}=0.2$ is a constant drag coefficient and $A_{b u b}$ is the bubble area.

The above form of the drag force is typically valid for only dilute systems but is satisfactory for the purposes of animation. We neglect the effect of the bubble momentum on the liquid phase since the bubble inertia is typically small. The coupling of the gas phase back to liquid phase and the use of drag laws that take account of large volume fractions of bubbles are both relatively straight forward to implement. The drag forces try to make the bubbles follow the complex flow patterns of the fluid. Drag force is typically size dependent and quadratic in velocity.

\subsection{Foam Model}

Bubbles that have risen to the liquid free surface naturally create dense foam structures. Typically this is in the form of a buoyant raft at the free surface of the fluid. A small volume of the foam raft is packed below the free surface creating a wet foam whilst most of the discrete bubbles are naturally lifted above the surface of the fluid and form a dry foam. The head on a glass of beer is a good example of such a foam raft.

We add a cohesive force $\mathbf{F}_{\mathbf{c o h}}$ between dry foam bubbles in order to produce realistic rheological behavior for the foam in which the bubbles naturally wish to aggregate. The form of the cohesive force used here is the same as for the collisional force $F_{n}$ except now the overlap is allowed to be negative in order to create a tensile force to hold the foam together. Thus the cohesion force when $\Delta x<0$ in equation 1 is:

$$
\mathbf{F}_{\text {coh }}=\mathbf{R F}_{\text {nt }}
$$

Bubble cohesion applies only to dry bubbles when they are above the surface of the fluid and not for free bubbles in the liquid. A breakage strength is assigned to such bonds so that sufficiently strong motion within the foam allows structural rearrangement of the bubbles and potentially fracture of the foam during stretching flow (such as over the sides of the beer glass). The cohesive force is also applied between dry bubbles and the solid surfaces (such as the beer glass) so that the foam can stick to these. If the bond strength is large then the foam is stronger and more rigid. If the bond strength is set to be low then a wetter more free flowing foam is created.

\subsection{Dissolved gas and transfer to discrete bubbles}

As described earlier, the source of bubbles in most drinks is dissolved gas. We need to track the gas concentration $G$ throughout the fluid and its transfer into the discrete bubbles. The gas transport is by diffusion and convection of the fluid. With the SPH method, the convection process is handled automatically. An SPH particle, representing a volume of fluid, carries its dissolved gas with it wherever it goes. This is a very attractive feature of SPH (and other Lagrangian methods) where we do not need to explicitly solve for the convective transport. The diffusive transport is governed by a conventional diffusion equation solved on the SPH particles. In SPH form the rate of change of gas concentration for particle $a$ is:

$$
\frac{\mathrm{d} G_{a}}{\mathrm{~d} t}=D \sum_{b} \frac{2 m_{b}}{\rho_{a} \rho_{b}} G_{a} \frac{\mathbf{r}_{a b} \cdot \nabla_{a} W_{a b}}{\mathbf{r}_{a b}^{2}+\eta^{2}}-N_{a}-E_{a}
$$

where $b$ is the particle under consideration and $a$ is the reference particle. $\mathbf{r}_{a b}$ is the position vector from particle $a$ to particle $b$. $W_{a b}=W\left(\mathbf{r}_{a b}, h\right)$ is the interpolation kernel evaluated for the distance $\left|\mathbf{r}_{a b}\right|$. Here $\eta$ is a small parameter used to smooth out the singularity at $\mathbf{r}_{a b}=0 . D$ is the diffusivity of the gas in the fluid, $N_{a}$ is the rate of gas loss due to nucleation and $E_{a}$ is the rate of gas loss from liquid due to expansion of nearby discrete bubbles.

A bubble is created from a nucleation site when enough gas has been transported by the fluid to the site to form a bubble of the minimum critical size (which can be specified by the user). Typical values would be of the order of $0.5 \mathrm{~mm}$ in diameter.

The transport of dissolved gas is a critical part of this model as it controls the rate of bubble generation. If the dissolved gas content is high or the liquid diffusivity is high, gas is transported to the nucleation site quickly and many bubbles are created. This is seen when fresh carbonated drink is first poured into a glass. As the dissolved gas is used, the bubble generation rate slows, since it takes longer for enough gas to be collected at the nucleation site in order to create the minimum bubble size. The sink term $N_{a}$ is given by subtracting the volume of gas entering the discrete bubble from the dissolved gas held by the SPH particle closest to the nucleation site.

When bubbles move, they grow by collecting more gas from the nearby liquid. This is also an inherently diffusion based process. The rate of increase of bubble volume is controlled by the amount of gas that is available in the close by SPH particles from which it can diffuse. The sink term $E_{a}$ in the dissolved gas diffusion equation is given by a simple mass balance removing the gas added to the discrete bubbles from the dissolved gas in the local SPH particles. This is an extremely important effect, since as the bubbles grow they rise faster due to the rapid increase in buoyancy versus drag 
force. This means bubbles become larger and more separated as they rise through the fluid.

\subsection{Bubble equations of motion}

The equations to be solved for each bubble are then just Newton's equation and a matching kinematic equation:

$$
\frac{\mathrm{d} \mathbf{v}_{\mathbf{a}}}{\mathrm{d} t}=\mathbf{F}_{\mathbf{b}}+\mathbf{F}_{\mathbf{d}}+\mathbf{F}_{\mathbf{c o l l}}+\mathbf{F}_{\mathbf{c o h}}
$$

and

$$
\frac{\mathrm{d} \mathbf{x}_{\mathbf{a}}}{\mathrm{d} t}=\mathbf{v}_{\mathbf{a}}
$$

These can be solved by any standard ODE integration scheme.

\subsection{Additional rules for the discrete bubbles}

Bubbles interact with other bubbles by collision. Collision can result in both coalescence and fragmentation of the bubbles into larger or smaller bubbles. These processes are included by adopting probabilistic rules during the collisions with the user choosing suitable values to achieve the type of behavior they are interested in. Bubbles within the dry foam are allowed to burst using a probabilistic model based on a specified half life for the bubbles.

\subsection{Summary of model attributes}

The rules used for discrete bubbles are summarized in Table 1 with

\begin{tabular}{|c|c|c|}
\hline Parameter & $\begin{array}{l}\text { Options and } \\
\text { Range }\end{array}$ & $\begin{array}{c}\text { Typical } \\
\text { value in } \\
\text { beer sim. }\end{array}$ \\
\hline Collision coefficient & $\begin{array}{l}0 \text { to } 1 . \text { Typical val- } \\
\text { ues: } 0.2 \text { to } 0.4\end{array}$ & 0.25 \\
\hline Collision spring stiffness & $\begin{array}{l}\text { Guided by bubble } \\
\text { mass }\end{array}$ & $0.01 \mathrm{~N} / \mathrm{m}$ \\
\hline Cohesion spring stiffness & $\begin{array}{l}\text { Guided by bubble } \\
\text { mass }\end{array}$ & $0.01 \mathrm{~N} / \mathrm{m}$ \\
\hline $\begin{array}{l}\text { Coalescence probability, } \\
\sec ^{-1}\end{array}$ & $\begin{array}{l}\text { Based on effect de- } \\
\text { sired }\end{array}$ & 0.0 \\
\hline $\begin{array}{l}\text { Splitting probability, } \\
\sec ^{-1}\end{array}$ & $\begin{array}{l}\text { Based on effect de- } \\
\text { sired }\end{array}$ & 0.0 \\
\hline $\begin{array}{l}\text { Bursting rate, half life, } \\
\text { sec }\end{array}$ & $\begin{array}{l}\text { Based on effect de- } \\
\text { sired }\end{array}$ & 0.0 \\
\hline $\begin{array}{l}\text { Initial gas fraction of liq- } \\
\text { uid }\end{array}$ & $\begin{array}{l}\text { Range from } 0.0 \text { to } \\
1.0\end{array}$ & 0.15 \\
\hline $\begin{array}{l}\text { Min. and max. nucle- } \\
\text { ation rad. }\end{array}$ & $\begin{array}{l}\text { Based on size } \\
\text { needed in applica- } \\
\text { tion }\end{array}$ & $0.4-0.5 \mathrm{~mm}$ \\
\hline $\begin{array}{l}\text { Nucleation density } \\
\left(/ \mathrm{cm}^{3}\right)\end{array}$ & Unlimited & 5.0 \\
\hline Gas diffusivity in liquid & $10^{-4}$ to 100 & 0.05 \\
\hline Bubble growth & $\begin{array}{l}\text { Bubbles absorb gas } \\
\text { from liquid and } \\
\text { grow if set to true } \\
\text { (true/false) }\end{array}$ & true \\
\hline
\end{tabular}
specific values used in the beer simulations.

Table 1: Summary of parameters, options and range available and values used for bubbles in the beer simulation.

In summary, this model for simulating bubbles accounts for all the key phenomenon observed during such processes. Our method of simulating bubbles as discrete entities coupled to both a particle based liquid phase and a dissolved gas phase gives us the capability of creating very realistic animations.

\section{Results}

The results presented in this section demonstrate specific aspects of the combined SPH-discrete bubble approach for modeling flow of bubbles in liquids. Rendered images of the dynamic bubble motion are shown in Figure 3. The object and the underlying fluid are not shown in this figure. Quite realistic motion of the bubble plumes and foam are seen here. In Figure 3(a), as the solid object moves to the right, the bubbles form a dense pattern around and especially underneath the object. This is due to the bubbles getting trapped below the object. As the object changes direction and moves to the left in Figure 3(b), the dense bubble structure now has freedom to move. It does so in the form of a plume emanating from the right of the object. There is a second stage of bubble accumulation occurring below the object at the same time at this stage. In Figure 3(c) the bubble plume now flows from the left of the object as the object moves to the right. The bubbles move to the surface of the liquid and form a layer of foam at this stage. The continuous supply of bubbles from the bottom leads to a steady increase in the thickness of the foam in Figure 3(d). The foam thickness is dependent on the balance between the rate of bubble arrival and the rate of bursting. These bubbles can coalesce or split during collision and can be sheared by the fluid.

The example we will use to demonstrate the capability of this model for creating realistic carbonated drink animations is the pouring of fresh beer into a beer glass. The glass begins empty and a stream of beer is poured into the glass. The surface of the beer glass provides the nucleation sites for bubble generation. The initial gas fraction of the beer was 0.15 . As the beer contacts the surface of the glass it creates plumes of bubbles which rise to create a foam head. By the end of the simulation when the glass is filled, around 180,000 SPH particles with a size of $4 \mathrm{~mm}$ have been created. By that time around 200,000 gas bubbles have also been created. The gas bubbles are nucleated at between 0.4 and $0.5 \mathrm{~mm}$. The bubbles reach a final maximum diameter of around $4.5 \mathrm{~mm}$ in the dry foam. This final maximum diameter is a function of the initial gas concentration in the liquid and rate of gas diffusion and can be selected to match the type of beverage that is required.

Figure 4 shows the sequence of steps for rendering of realistic looking simulated bubble flow. The frame shown here is mid way through the filling of the glass. The first image (left) shows the liquid phase by itself flowing into a beer mug. The second image shows just the bubbles that have been generated in the beer glass by this time. These are rendered as spheres with Maya software shading. The last image in Figure 4 shows the liquid with the bubbles composited.

A final rendered image sequence of beer animation is presented in Figure 5. Images on the top represent the beer rendered as ale and the images on the bottom show the beer rendered as stout. The rendering was performed via Maya using Mentalray renderer with standard reflection and refraction used for the glass. Independent renders for the beer mug, liquid and bubbles were performed. Compositing of these different parts of the render was achieved using the software After Effect. Rendering in separate layers allowed more control over specific elements of the simulation while compositing.

The fresh beer in the free stream does not initially contain any bubbles. As the beer hits the glass surface a strong plume of bubbles is generated which rises rapidly to the surface creating an initial head on the beer. The incoming beer stream creates strong swirling recirculation in the glass. Many bubbles are dragged around with the swirling fluid so that the beer is fairly cloudy. As the filling of the glass continues the beer head expands as the clouds of bubbles rise through the beer. In the third frame, the glass is more than half filled with much of the beer becoming clear of bubbles and is now trans- 


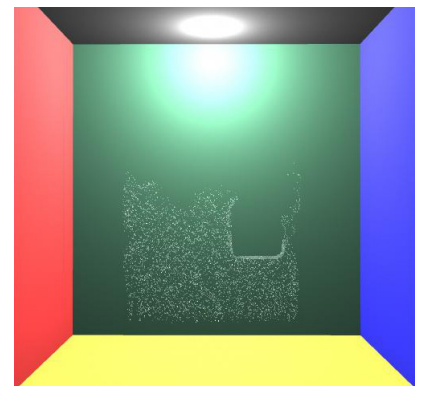

(a)

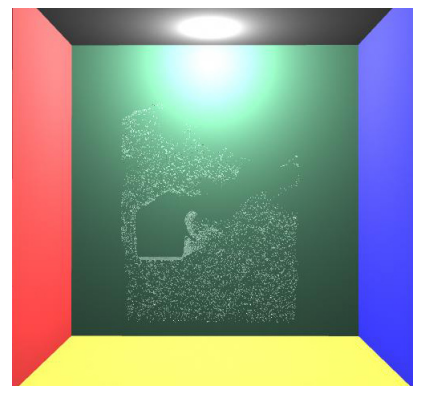

(b)

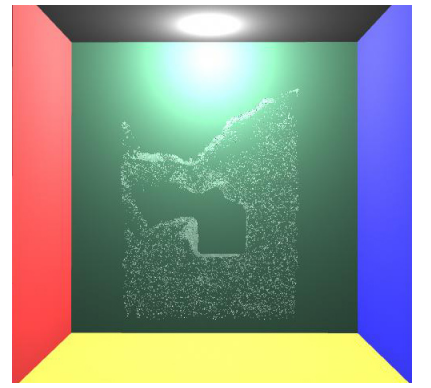

(c)

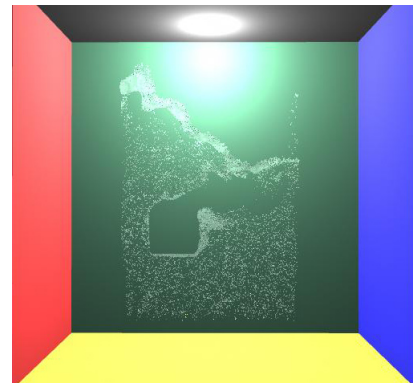

(d)

Figure 3: Dynamic bubble behavior:(a) $t=0.5$, (b) $t=1.0$, (c) $t=1.5$, (d) $t=2.0$ s. The liquid and moving object are not shown. Bubbles are randomly generated from the tank bottom. The square object translates across the tank.
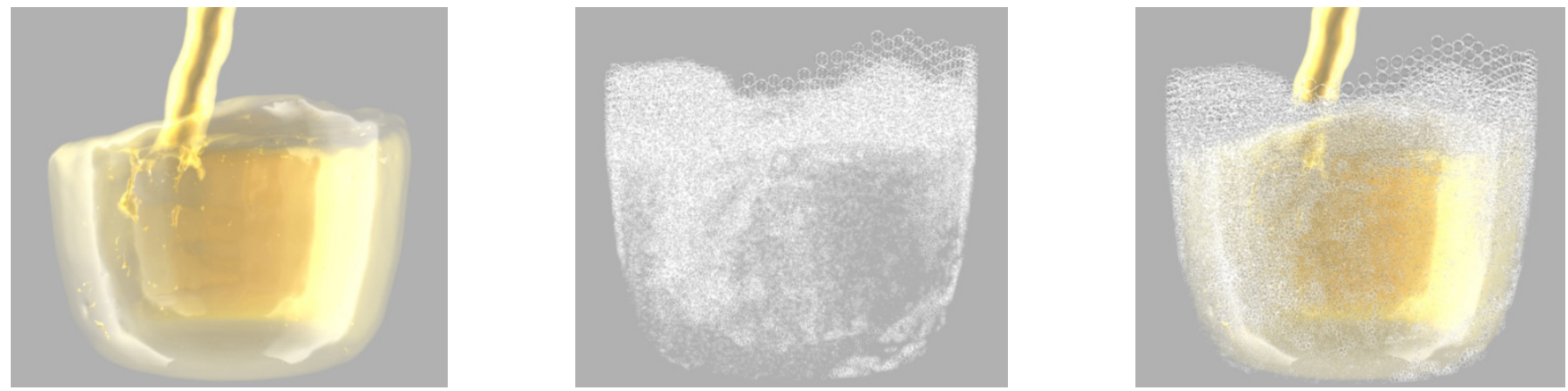

Figure 4: Towards realistic beer simulation: First image shows the liquid phase, the second image shows the bubble phase and the third image shows the liquid and bubbles composited forming a realistic beer model.

parent. A strong cloud of bubbles continues to rise on the left where the fresh beer is entering and contacting the glass near the bottom. The generation of the bubble plume from the newly entered beer is a very realistic feature of this model. For the ale version, the beer pouring is stopped before the final frame and new bubble generation slows considerably. Most bubbles have now joined the head of the beer with a dilute band of slower fine bubbles still rising in the upper third of the beer.

In the stout, different bubble controls were chosen so as to slow the initial surge of bubbles leading to a slower growth of the head on the beer. The pouring of the stout is continued for longer so that the glass fills completely and a thicker head is formed all the way to the lip of the glass. Since the stout is more opaque the internal distribution of the bubble plumes is less visible. In both cases, the fluid filling, the internal distributions of the bubbles, the timing of the bubble creation and formation and structure of the beer froth are quite realistic. This form of model can be used for other beverages and many other forms of fluid-bubble flows that are needed for digital content.

Animations of the above can be found at:

http://www.cmis.csiro.au/cfd/sph_movies

\section{Conclusion}

We have presented a new coupled discrete bubble-SPH based simulation technique to automatically and naturally animate bubbly fluid flows such as beer, champagne and carbonated drinks. The bubbles are represented as discrete spherical bodies and are coupled to the fluid flow. The generation of the bubbles is driven by predictions of diffusion of dissolved gas content and its transfer via nucleation processes into the discrete bubbles. This provides natural and realistic rates of bubble generation in different regions of the flow. Ad- ditionally bubbles are able to grow as they rise in the fluid and form stable foams or froths with plausible rheological behavior. Bubble collisions can include coalescence and fragmentation and foam dissipation can also be controlled easily. This modeling approach includes a significant amount of real physics which enables users to create a broad range of bubble-fluid effects without having to specify large numbers of artificial controls. The use of the nucleation and dissolved gas diffusion physics means that a few simple real physical quantities can be used to control the complex processes involved in these flows. In this paper, we have only focused our attention on bubbles generated from gas in liquid for carbonated liquids. However the same physical processes control many other bubble-fluid flows enabling this method to be applied more broadly.

\section{Acknowledgments}

The authors would like to thank "theLabSydney" Australia for their contribution towards rendering the realistic beer simulations. This research was partly supported by the Korea Ministry of Information and Communication.

\section{References}

Cleary, P. W., ANd PraKash, M. 2004. Discrete element modelling and smooth particle hydrodynamics: potential in the environmental sciences. Phil. Trans. Roy. Soc. A 362, 2003-2030.

Cleary, P., Prakash, M., Ha, J., Stokes, N., and Scott, C. 2007. Smoothed particle hydrodynamics: Status and future potential. Progress in Computational Fluid Dynamics 7, 70-90.

Cleary, P. W. 1998. Modelling confined multi-material heat and mass flows using SPH. App. Math. Modelling 22, 981-993. 

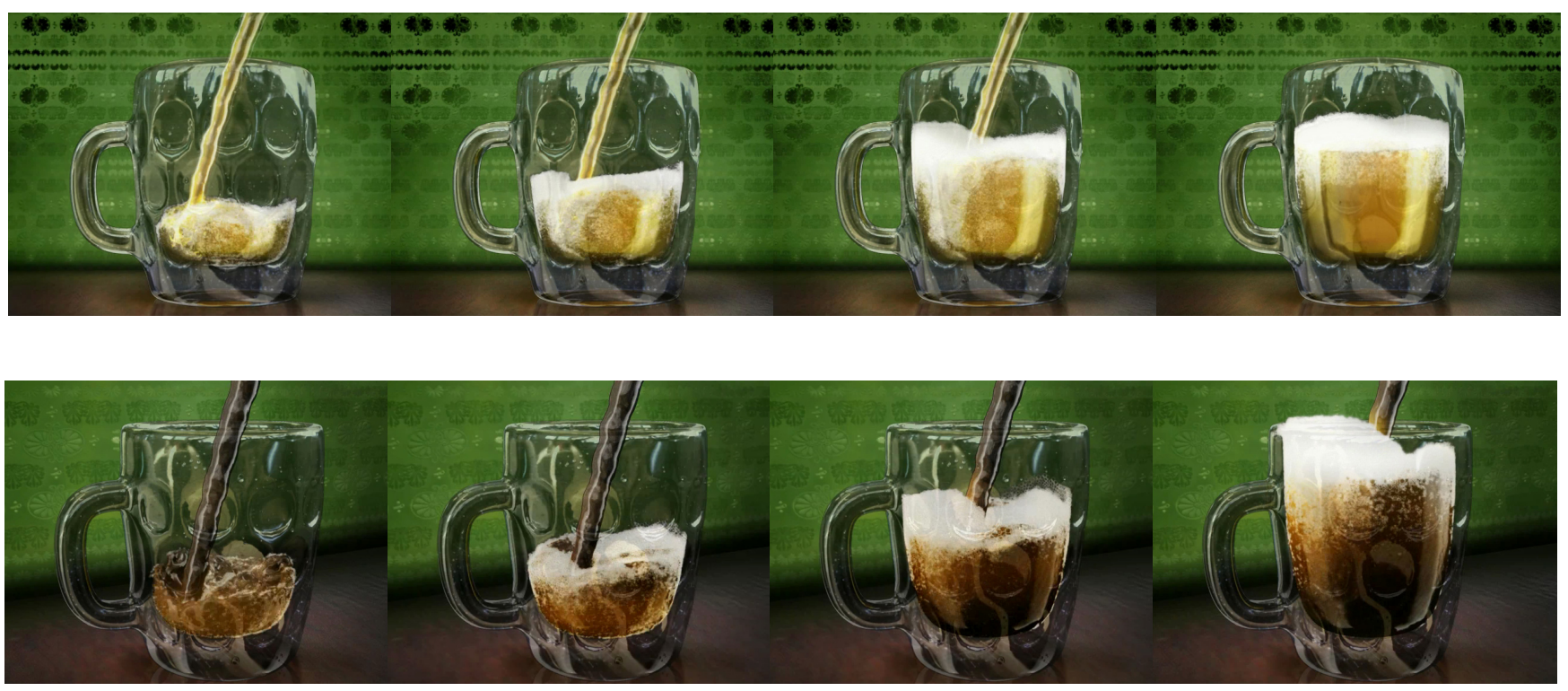

Figure 5: Simulation of pouring of Ale and Stout into a beer mug: Images on the top show realistic rendered images of Ale and the bottom images indicate realistic rendered images of Stout

Cleary, P. W. 2004. Large scale industrial DEM modelling. Engineering Computations 21, 169-204.

Desbrun, M., And Gascuel, M. P. 1996. Smoothed particles: A new paradigm for animating highly deformable bodies. In Proceedings of SCA 1996, Springer-Verlag, R. Boulic and G. Hegron, Eds., 61-76.

EnRIGHT, D., MARschner, S., AND FEDKIW, R. 2002. Animation and rendering of complex water surfaces. In Proceedings of ACM SIGGRAPH 2002, ACM Press, 736-744.

Foster, N., AND FedKIW, R. 2001. Practical animation of liquids. In Proceedings of ACM SIGGRAPH 2001, ACM Press, 23-30.

Foster, N., AND Metaxas, D. 1996. Realistic animation of liquids. Graph. Models Image Process. 58, 5, 471-483.

Geiger, W., Leo, M., Rasmussen, N., Losasso, F., And FEDKIW, R. 2006. So real it'll make you wet. Proceedings of ACM SIGGRAPH 2006 Sketch.

Gingold, R. A., And Monaghan, J. J. 1977. Smoothed particle hydrodynamics: Theory and application to non-spherical stars. Mon. Not. Roy. Astron. Soc. 181, 375-389.

Greenwood, S. T., And House, D. H. 2004. Better with bubbles: enhancing the visual realism of simulated fluid. In Proceedings of SCA 2004, ACM Press, 287-296.

Hong, J.-M., AND Kim, C.-H. 2003. Animation of bubbles in liquid. Computer Graphics Forum 22, 3, 253-262.

Hong, J.-M., AND KIM, C.-H. 2005. Discontinuous fluids. In Proceedings of ACM SIGGRAPH 2005, ACM Press, 915-920.

KajiYA, J. T., And Herzen, B. V. 1984. Ray tracing volume densities. Proceedings of ACM SIGGRAPH 1984 18, 3, 165174.

Kim, J., Cha, D., Chang, B., Koo, B., and Ihm, I. 2006. Practical animation of turbulent splashing water. In Proceedings of SCA 2006, Eurographics Association, 335-344.

Kuck, H., Vogelgsang, C., And Greiner, G. 2002. Simulation and rendering of liquid foams. Graphics Interface, 81-88.
Mihalef, V., Unlusu, B., Sussman, M., and Metaxas, D. 2006. Physics-based boiling simulation. In Proceedings of SCA 2006, Eurographics Association, 317-324.

Monaghan, J. J. 1992. Smoothed particle hydrodynamics. Ann. Rev. Astron. Astrophys. 30, 543-574.

Monaghan, J. J. 1994. Simulating free surface flows with SPH. J. Comp. Phys. 110, 399-406.

Monaghan, J. J. 2005. Smoothed particle hydrodynamics. Report on Progress in Physics 68, 1703-1759.

Muller, M., Charypar, D., And Gross, M. 2003. Particlebased fluid simulation for interactive applications. In Proceedings of SCA 2003, Eurographics Association, 154-159.

Muller, M., Solenthaler, B., Keiser, R., And Gross, M. 2005. Particle-based fluid-fluid interaction. In Proceedings of SCA 2005, ACM Press, 237-244.

Premoze, S., TAsdizen, T., Bigler, J., Lefohn, A., AND WhitAKer, R. T. 2003. Particle-based simulation of fluids. Computer Graphics Forum 22, 3.

Song, O.-Y., SHIN, H., AND Ko, H.-S. 2005. Stable but nondissipative water. In Proceedings of ACM SIGGRAPH 2005, ACM Press, 81-97.

STAM, J. 1999. Stable fluids. In Proceedings of ACM SIGGRAPH 1999, ACM Press/Addison-Wesley Publishing Co., 121-128.

Takahashi, T., FujiI, H., Kunimatsu, A., Hiwada, K., SAITO, T., TANAKA, K., AND UEKI, H. 2003. Realisitc animation of fluid with splash and foam. Computer Graphics Forum $22,3,391-400$.

Thürey, N., Rude, U., And Stamminger, M. 2006. Animation of open water phenomena with coupled shallow water and free surface simulations. In Proceedings of SCA 2006, Eurographics Association, 157-166.

WANG, H., Mucha, P. J., AND TURK, G. 2005. Water drops on surfaces. In Proceedings of ACM SIGGRAPH 2005, ACM Press, 921-929.

Zheng, W., Yong, J.-H., AND PaUl, J.-C. 2006. Simulation of bubbles. In Proceedings of SCA 2006, Eurographics Association, 325-333. 